\title{
Genome-wide analysis of single-locus and epistasis single-nucleotide polymorphism effects on anti-cyclic citrullinated peptide as a measure of rheumatoid arthritis
}

\author{
Li Ma, Daniel Dvorkin, John R Garbe and Yang Da*
}

Address: Department of Animal Science, University of Minnesota, 1364 Eckles Avenue, St. Paul, Minnesota 55108, USA

Email: Li Ma - maxxx131@umn.edu; Daniel Dvorkin - danield@biostat.umn.edu; John R Garbe - jgarbe@umn.edu; Yang Da* - yda@umn.edu

* Corresponding author

from Genetic Analysis Workshop 15

St. Pete Beach, Florida, USA. II-15 November 2006

Published: 18 December 2007

BMC Proceedings 2007, I(SuppI I):SI27

This article is available from: http://www.biomedcentral.com/I753-656I/I/SI/SI27

(C) 2007 Ma et al; licensee BioMed Central Ltd.

This is an open access article distributed under the terms of the Creative Commons Attribution License (http://creativecommons.org/licenses/by/2.0), which permits unrestricted use, distribution, and reproduction in any medium, provided the original work is properly cited.

\begin{abstract}
The goal of this study was to identify single-locus and epistasis effects of SNP markers on anti-cyclic citrullinated peptide (anti-CCP) that is associated with rheumatoid arthritis, using the North American Rheumatoid Arthritis Consortium data. A square root transformation of the phenotypic values of anti-CCP with sex, smoking status, and a selected subset of 20 single-nucleotide polymorphism (SNP) markers in the model achieved residual normality $(p>0.05)$. Three singlelocus effects of two SNPs were significant $\left(p<10^{-4}\right)$. The epistasis analysis tested five effects of each pair of SNPs, the two-locus interaction, additive $\times$ additive, additive $\times$ dominance, dominance $x$ additive, and dominance $x$ dominance effects. A total of ten epistasis effects of eight pairs of SNPs on II autosomes and the $X$ chromosome had significant epistasis effects $\left(p<10^{-7}\right)$. Three of these epistasis effects reached significance levels of $p<10^{-8}, p<10^{-9}$, and $p<10^{-10}$, respectively. Two potential SNP epistasis networks were identified. The results indicate that the genetic factors underlying anti-CCP may include single-gene action and gene interactions and that the geneinteraction mechanism underlying anti-CCP could be a complex mechanism involving pairwise epistasis effects and multiple SNPs.
\end{abstract}

\section{Background}

The data set of the North American Rheumatoid Arthritis Consortium (NARAC) for Genetic Analysis Workshop 15 (GAW15) contains genotypes of 5700 SNPs covering all 23 human chromosomes, affected status of rheumatoid arthritis (RA), and a number of quantitative traits including anti-cyclic citrullinated peptide (anti-CCP). Anti-CCP is associated with RA and is used by some as a measure of
RA [1]. Linkage analysis of the RA status in the NARAC data using affected sib-pair method has been reported [2].

\section{Methods}

The NARAC data set was edited by requiring each individual to have SNP genotypes on the 5700 SNPs and antiCCP record, and 1466 individuals satisfied this criterion. The anti-CCP values significantly deviated from normal distribution with $p<0.01$ (Fig. $1 \mathrm{~A}$ ), according to the Sha- 
piro-Wilk, Kolmogorov-Smirnov, Cramer-von Mises, and Anderson-Darling tests offered by SAS UNIVARIATE PROCEDURE [3]. Because the residual normal distribution, not the phenotypic normal distribution, is required for the statistical tests in this article, a statistical model that achieves residual normality was found using the procedure leading to Figures $1 \mathrm{~B}-1 \mathrm{~F}$. The untransformed antiCCP did not achieve residual normality $(p<0.01$; Fig. $1 \mathrm{~B}-$ 1C). The Box-Cox transformation [4] for a range of $\lambda$ values and the square root transformation of anti-CCP were evaluated to find an optimal transformation that has the minimal sum of squares under the model for Figure 1B and improves the residual normality. None of the transformations achieved residual normality $(p<0.01)$, but the square root transformation was found to have minimal residual sum of squares. The residual distribution under this transformation is shown in Figure 1D. For the model used in Figure 1D, a total of 41 SNPs were found to have significant single-locus and epistasis effects with the same significance level as the SNPs for Figure 1C. Adding all the 41 SNPs in the model for Figure 1D achieved a near-perfect residual normality $(p>0.15$; Fig. $1 \mathrm{E})$. To reduce the model degrees of freedom or increase the residual degrees of freedom, step-wise elimination of SNPs from the full model for Figure 1E was conducted to find the minimal set of SNPs that had 20 SNPs (Table 1) and still achieved residual normality for the transformed data $(p>0.05$; Fig. $1 F)$. In the model for Figure $1 F$, each SNP with a singlelocus effect was fitted in model as a locus with three genotypes while each pair of SNPs were fitted in the model as a genetic factor with nine $(3 \times 3)$ genotypes. Each SNP or SNP pair in this subset was re-tested by treating the other SNPs in the set as fixed effects (in addition to sex and smoking status). For all SNPs not in this subset, the SNP effects were tested based on the model for Figure 1F. The model for testing single-locus effects was

$$
(y)^{1 / 2}=s e x+C i g E+(\text { normality SNPs })+S N P+e,
$$

where $(y)^{1 / 2}$ is the square root transformed anti-CCP, sex is the gender of the individual, $\mathrm{CigE}$ is the indicator variable whether the person ever smoked, (normality SNPs) is the 20 SNPs in Table 1 to achieve residual normality shown in Figure $1 \mathrm{~F}, \mathrm{SNP}$ is the SNP being tested for three singlelocus effects (the SNP marker effect, and additive and dominance effects), and $e$ is the random residual. The significance test of the SNP marker effect used an F-test, and $t$-tests were used to test additive and dominance effects. The epistasis analysis tested five effects of each pair of SNPs: two-locus interaction (I), additive $\times$ additive $(\mathrm{A} \times$ $A)$, additive $\times$ dominance $(A \times D)$, dominance $\times$ additive $(\mathrm{D} \times \mathrm{A})$, and dominance $\times$ dominance $(\mathrm{D} \times \mathrm{D})$ epistasis effects. The genetic interpretation of the $\mathrm{A} \times \mathrm{A}, \mathrm{A} \times \mathrm{D}, \mathrm{D} \times$ $\mathrm{A}$, and $\mathrm{D} \times \mathrm{D}$ epistasis effects are allele $\times$ allele, allele $\times$ genotype, genotype $\times$ allele, and genotype $\times$ genotype interactions, respectively. The model for testing epistasis effects was

$$
(y)^{1 / 2}=\text { sex }+ \text { CigE }+(\text { normality SNPs })+S N P 1+S N P 2+
$$$$
S N P 1 * S N P 2+e \text {, }
$$

where $S N P 1{ }^{*} S N P 2$ is the interaction effect between the two SNPs, to be denoted by " $I$ ". The significance test of the $I$-effect used an F-test, and $t$-tests were used to test four individual epistasis effects, $\mathrm{A} \times \mathrm{A}, \mathrm{A} \times \mathrm{D}, \mathrm{D} \times \mathrm{A}$, and $\mathrm{D} \times$ $\mathrm{D}$, using an extended Kempthorne model that allows Hardy-Weinberg and linkage disequilibria [5]. The I-effect

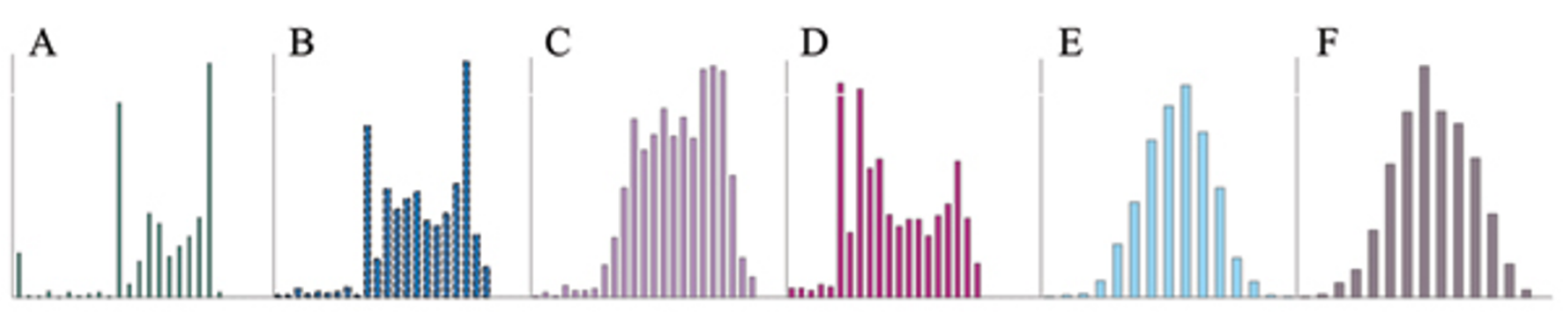

\section{Figure I}

Histograms of anti-CCP distributions. A-D significantly deviated from the normal distribution $(p<0.0 \mathrm{I})$. The deviations from normal distribution were insignificant in $E(p>0.15)$ and $F(p>0.05)$. A, Phenotypic distribution of anti-CCP values; $B$, residual distribution of anti-CCP values under the model with sex and smoking status as the fixed effects; $C$, residual distribution of anti-CCP values under the model with sex, smoking status, and all 37 SNPs with significant single-locus and epistasis effects as the fixed effects; $D$, residual distribution of the square root transformed anti-CCP values under the model with sex and smoking status as the fixed effects; E, residual distribution of the square root transformed anti-CCP values under the model with sex, smoking status, and all 4I SNPs with significant single-locus and epistasis effects as the fixed effects; F, residual distribution of the square root transformed anti-CCP values under the model with sex, smoking status, and a minimal subset of 20 SNPs from the 4I SNPs in E, to achieve normality. 
Table I: Minimal set of SNPs to achieve residual normality of the square root transformed anti-CCP values in Figure IF

\begin{tabular}{|c|c|c|c|}
\hline Chromosome 2 & SNPI & Chromosome 2 & SNP2 \\
\hline 2 & rs2685263 & $\mathrm{a}^{\mathrm{a}}$ & - \\
\hline 6 & rs 1892512 & - & - \\
\hline 2 & rs289847 & 16 & rs9033 \\
\hline 2 & rs981395 & 23 & rsII08444 \\
\hline 3 & rsI3975 & 5 & rs1016256 \\
\hline 6 & rs|398576 & 18 & rs884205 \\
\hline 8 & rsl000236 & 9 & rs75I340 \\
\hline 12 & rs|400|42 & 14 & rs 1959068 \\
\hline 12 & rs|843910 & 22 & rs743409 \\
\hline 14 & rs1033594 & 17 & rs759563 \\
\hline 16 & rs 194790 & 17 & rs 1872076 \\
\hline
\end{tabular}

a-, The left marker had a significant single-locus effect.

answers the question whether the two loci had an interaction whereas an individual epistasis effect identifies the exact mode of the interaction. For testing epistasis effects involving the $\mathrm{X}$ chromosome, only females were included in the analysis. The single-locus and epistasis tests using Models (1-2) were implemented using the epiSNP computer package developed by the authors [6].

\section{Results}

Significant single-locus SNP effects

The single-locus analysis using Model (1) had 17,100 tests for 5700 SNPs. Two SNPs on chromosomes 2 and 3 had three significant effects on the transformed data with $p<$ $10^{-4}$ (Table 2). With the stringent significance level of $p<$ $10^{-4}, 17,100$ tests are expected to yield 1.7 significant results by chance. Therefore, the three significant effects of two SNPs could be due to chance. Results with less stringent significance levels may be of interest to compare with reported results for possible confirmations. A total of 231 single-locus effects of all chromosomes were significant at $p<0.01$.

\section{Significant epistasis effects}

The epistasis analysis using Model (2) had 81,210,750 tests for 5700 SNPs. Ten significant epistasis effects of eight pairs of SNPs on 11 autosomes and the X chromosome reached the significance level of $p<10^{-7}$ (Table 3 ). Of these ten effects, three effects of two pairs of SNPs reached significance levels of $p<10^{-8}, p<10^{-9}$, and $p<10^{-}$ 10 , respectively (Table 3 ). The most significant epistasis effect was an additive $\times$ additive effect between two SNPs on chromosome 11 and the $\mathrm{X}$ chromosome, followed by a dominance $\times$ additive effect between chromosomes 7 and 10 . With the significance level of $p<10^{-7}$, the expected number of significant results out of $81,210,750$ tests due to chance is eight, so that ten observed significant epistasis effects were more than expected by chance. More importantly, the three significant epistasis effects with $p<10^{-8}$, $p$ $<10^{-9}$, and $p<10^{-10}$ far exceeded the significance level defined by the threshold $p$-value of $10^{-7}$ to be considered as random results. Based on this analysis, evidence for epistasis effects was stronger than single effects reported in this article.

\section{Complex gene interaction mechanism and epistasis network}

The epistasis results indicate that the gene interaction mechanism underlying anti-CCP could be a complex mechanism involving three types of epistasis effects and multiple SNPs, where 'three types of epistasis' refers to A $\times$ $\mathrm{A}, \mathrm{A} \times \mathrm{D}$ or $\mathrm{D} \times \mathrm{A}$, and $\mathrm{D} \times \mathrm{D}$. The exact content of this complex mechanism can be illustrated using the example of three highly significant pairs of SNPs, rs 1009172 (chromosome 11) and rs17410 (chromosome X), rs2056553 (chromosome 7) and rs1328327 (chromosome 10), and rs1400142 (chromosome 12) and rs1959068 (chromosome 14). The exact gene interaction mechanism of these three pairs is $(A \times A)-(D \times A)-(D \times D)$. Close examination of each of the three epistasis effects showed that individuals with 1_1 allele combination of rs1009172 (chromosome 11) and rs17410 (chromosome X) SNP pair, 22_2 genotype-allele combination of rs2056553 (chromosome

Table 2: Significant single-locus SNP effects on square root transformed anti-CCP with $p<10^{-4}$

\begin{tabular}{lcccc}
\hline Chromosome & SNP & Chromosome location (bp) & Effect & $p$-Value \\
\hline 2 & rsl017267 & 539,873 & Marker & $0.525 \times 10^{-4}$ \\
2 & rs 1017267 & 539,873 & Dominance & $0.403 \times 10^{-4}$ \\
3 & rsI401337 & $149,511,657$ & Marker & $0.628 \times 10^{-4}$ \\
\hline
\end{tabular}


Table 3: Significant SNP epistasis effects on square root transformed anti-CCP with $p<10^{-7}$

\begin{tabular}{|c|c|c|c|c|c|}
\hline Chromosome I & SNPI & Chromosome 2 & SNP2 & Effect $^{\mathrm{a}}$ & $p$-Value \\
\hline I & rs2031750 & 18 & rs1395610 & $A \times A$ & $0.827 \times 10^{-7}$ \\
\hline 2 & rs/26/238 & 23 & rs953II4 & $D \times A$ & $0.976 \times 10^{-7}$ \\
\hline 6 & rs310389 & 13 & rs494I527 & I & $0.563 \times 10^{-7}$ \\
\hline 6 & rs310389 & 13 & rs494I527 & $D \times A$ & $0.765 \times 10^{-7}$ \\
\hline 6 & rs873460 & 16 & rs874562 & $A \times A$ & $0.754 \times 10^{-7}$ \\
\hline 6 & rsI087924 & 23 & rs644429 & I & $0.583 \times 10^{-7}$ \\
\hline 7 & rs2056553 & 10 & rs 1328327 & $D \times A$ & $0.583 \times 10^{-8}$ \\
\hline 11 & rs1009172 & 23 & rs 17410 & I & $0.612 \times 10^{-9}$ \\
\hline 11 & rs1009172 & 23 & rs 17410 & $A \times A$ & $0.247 \times 10^{-10}$ \\
\hline 12 & rs|400I42 & 14 & rs1959068 & $D \times D$ & $0.437 \times 10^{-7}$ \\
\hline
\end{tabular}

al, two-locus interaction effect; $A \times A$, additive $\times$ additive effect; $A \times D$, additive $\times$ dominance effect; $D \times A$, dominance $\times$ additive effect; $D \times D$, dominance $\times$ dominance effect.

7) and rs1328327 (chromosome 10) SNP pair, and 11_12 genotype-genotype combination of rs 1400142 (chromosome 12) and rs1959068 (chromosome 14) SNP pair had the lowest square root transformed anti-CCP values. In contrast, individuals with 1_2 allele combination of rs1009172 (chromosome 11) and rs17410 (chromosome $\mathrm{X})$ SNP pair, 12_2 genotype-allele combination of rs2056553 (chromosome 7) and rs1328327 (chromosome 10) SNP pair, and 11_11 genotype-genotype combination of rs1400142 (chromosome 12) and rs1959068 (chromosome 14) SNP pair had the highest square root transformed anti-CCP values. In addition to highly significant epistasis effects, an epistasis network around a locus that has a highly significant epistasis effect with at least one locus and interacted with a large number of other loci at a lower significance level (higher $p$-value) should be of interest, because many significant epistasis effects involving one locus at a lower significance level are less likely to be random than a single epistasis effect at the same significance level. Figure 2 shows two examples of such epistasis network.

\section{Discussion}

The results showed that evidence for epistasis effects was stronger than for single-locus effects on anti-CCP. This implies that gene interactions could be an important
(A)

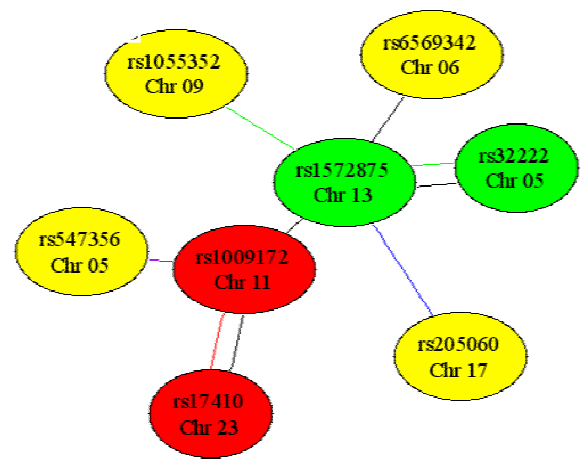

(B)

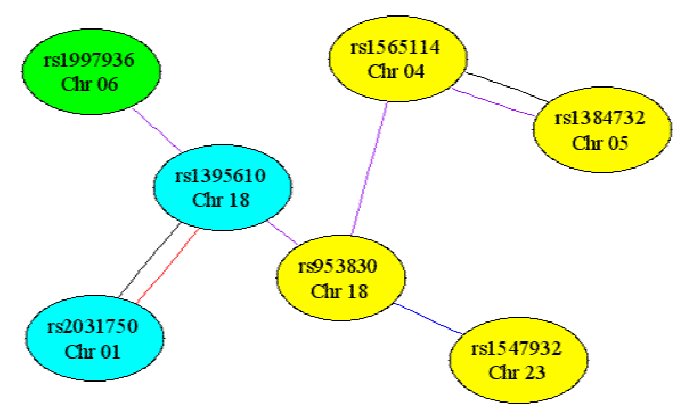

Figure 2

Two potential epistasis networks each centered at an SNP that had one of the most significant epistasis effects and interacted with other loci at lower significance levels. Each type of epistasis effect is represented by the line color: black, I effect; red, $A \times A$; purple, $A \times D$; blue, $D \times A$; green, $D \times D$. A pair of SNPs connected by two lines had two significant epistasis effects. The significance level of each epistasis effect is represented by the node color: red, $p<10^{-10}$; cyan, $p<10^{-7}$; green, $p<10^{-6}$; yellow, $p<10^{-5}$. A, Epistasis network centered at rs I009I 72 of chromosome II; B, Epistasis network centered at rs 1395610 of chromosome 18 . 
genetic factor underlying rheumatoid arthritis. The phenotypic distribution of anti-CCP might merit further study to identify factors that caused the distribution curve with multiple peaks as shown in Figure 1A. Including such factors in the statistical model could achieve residual normality without using as many 'normality SNPs' and hence increases the residual degrees of freedom.

\section{Conclusion}

The genetic factors underlying anti-CCP may include single-gene action and gene interactions but gene interaction effects could be more important than single-gene effects. The gene interaction mechanism could be a complex mechanism involving a number of SNPs and three types of pairwise epistasis effects, and the epistasis results could be used to identify allelic and genotypic combinations with the highest and lowest anti-CCP levels.

\section{Competing interests}

The author(s) declare that they have no competing interests.

\section{Acknowledgements}

This article has been published as part of BMC Proceedings Volume I Supplement I, 2007: Genetic Analysis Workshop 15: Gene Expression Analysis and Approaches to Detecting Multiple Functional Loci. The full contents of the supplement are available online at http://www.biomedcentral.com/ $\underline{|753-656| / \mid \text { ? issue=SI. }}$.

\section{References}

I. Firestein GS: Evolving concepts of rheumatoid arthritis. Nature 2003, 423:356-36I.

2. Amos Cl, Chen WV, Lee A, Li W, Kern M, Lundsten R, Batliwalla F, Wener M, Remmers E, Kastner DA, Criswell LA, Seldin MF, Gregersen PK: High-density SNP analysis of $\mathbf{6 4 2}$ Caucasian families with rheumatoid arthritis identifies two new linkage regions on IIpI 2 and 2q33. Genes Immunol 2006, 7:277-286.

3. SAS Institute Inc: SAS User's Guide. Cary, North Carolina: SAS Institute, Inc; 1990.

4. Box GEP, Cox DR: An analysis of transformations. J Roy Stat Soc Ser B 1964, 26:2II-246.

5. Mao Y, London NR, Ma L, Dvorkin D, Da Y: Detection of SNP epistasis effects of quantitative traits using an extended Kempthorne model. Physiol Genomics 2006, 28:46-52.

6. Ma L, Dvorkin D, Garbe JR, Da Y: epiSNP User Manual Version I.I w. [http://animalgene.umn.edu/episnp/index.html]. Department of Animal Science, University of Minnesota
Publish with Bio Med Central and every scientist can read your work free of charge

"BioMed Central will be the most significant development for disseminating the results of biomedical research in our lifetime. " Sir Paul Nurse, Cancer Research UK

Your research papers will be:

- available free of charge to the entire biomedical community

- peer reviewed and published immediately upon acceptance

- cited in PubMed and archived on PubMed Central

- yours - you keep the copyright

Submit your manuscript here:

http://www.biomedcentral.com/info/publishing_adv.asp 